\title{
An Analysis of the Effect of Noise in a Heterogeneous Agent Financial Market Model *
}

\author{
Carl Chiarella ${ }^{1}$, Xue-Zhong $\mathrm{He}^{1}$ and Min Zheng ${ }^{1,2, * *}$ \\ ${ }^{1}$ School of Finance and Economics, University of Technology, Sydney \\ PO Box 123, Broadway, NSW 2007, Australia \\ carl.chiarella@uts.edu.au, tony.he1@uts.edu.au, min.zheng@uts.edu.au \\ ${ }^{2}$ School of Mathematical Sciences, Peking University \\ Beijing 100871, P. R. China
}

July 30, 2009

\begin{abstract}
Heterogeneous agent models (HAMs) in finance and economics are often characterised by high dimensional nonlinear stochastic differential or difference systems. Because of the complexity of the interaction between the nonlinearities and noise, a commonly used indirect approach to the study of HAMs combines theoretical analysis of the underlying deterministic skeleton with numerical analysis of the stochastic model. However, a natural question to ask is whether this indirect approach properly characterises the nature of the stochastic model. This paper aims to tackle this question by developing a direct and analytical approach to the analysis of a stochastic model of speculative price dynamics involving two types of agents, fundamentalists and chartists, and the market price equilibria of which can be characterised by the stationary measures of a stochastic dynamical system. Using the stochastic method of averaging, we show that the stochastic model displays behaviour consistent with that of the underlying deterministic model when the time lag in the formation of the price trends used by the chartists is not too close to zero. However, when this lag approaches zero, such consistency breaks down.
\end{abstract}

Key Words: Heterogeneous agents, speculative behaviour, stochastic bifurcations, stationary measures, chartists.

*This work was initiated while Zheng was visiting the Quantitative Finance Research Centre (QFRC) at the University of Technology, Sydney (UTS), whose hospitality she gratefully acknowledges. The work reported here has received financial support from the Australian Research Council (ARC) under a Discovery Grant (DP0450526), UTS under a Research Excellence Grant, and a National Science Foundation Grant of China (10871005).

** Corresponding author. 


\section{Introduction}

Traditional economic and finance theory based on the paradigm of the representative agent with rational expectations has not only been questioned because of the strong assumptions of agent homogeneity and rationality, but has also encountered some difficulty in explaining the market anomalies and stylised facts that show up in many empirical studies, including high trading volume, excess volatility, volatility clustering, long-range dependence, skewness, and excess kurtosis (see Pagan (1996) and Lux (2009) for a description of the various anomalies and stylised facts). As a result, there has been a rapid growth in the literature on heterogeneous agent models that is well summarised in the recent survey papers by Hommes (2006), LeBaron (2006), Hommes and Wagener (2009) and Chiarella, Dieci and He (2009). These models characterise the dynamics of financial asset prices and returns resulting from the interaction of heterogeneous agents having different attitudes to risk and having different expectations about the future evolution of prices. For example, Brock and Hommes (1997, 1998) propose a simple Adaptive Belief System to model economic and financial markets. A key aspect of these models is that they exhibit feedback of expectations. The resulting dynamical system is nonlinear and, as Brock and Hommes (1998) show, capable of generating complex behaviour from local stability to (a)periodic cycles and even chaos. By adding noise to the underlying deterministic system and using the simulation approach, many models (see, for example, Hommes (2002), Chiarella, He and Hommes (2006a, 2006b)) are able to generate realistic time series. In particular, it has been shown (see for instance Hommes (2002), He and Li (2007) and Lux (2009)) that such simple nonlinear adaptive models are capable of capturing important empirically observed features of real financial time series, including fat tails, clustering in volatility and power-law behaviour (in returns). Most of the stylised simple evolutionary adaptive models that one encounters in the literature are analysed within a discrete-time framework and their numerical analysis provides insights into the connection between individual and market behaviour.

One of the most important issues for many heterogeneous agent asset pricing models is the interaction of the behaviour of the heterogeneous agents and the interplay of noise with the underlying nonlinear deterministic market dynamics. Indeed He and Li (2007) in their simulations find that these two effects interact in ways which are not yet understood at a theoretical level. The noise can be either fundamental noise or market noise, or both. The commonly-used approach (except the stochastic approach developed in Lux (1995, 1997, 1998)), referred to as the indirect approach for convenience, is first to consider the corresponding deterministic "skeleton" of the stochastic model where the noise terms are set to zero and to investigate the dynamics of this nonlinear deterministic system by using stability and bifurcation theory; one then uses simulation methods to examine the interplay of various types of noise with the deterministic dynamics. This approach relies on a combination of simulations and faith that the properties of the deterministic system carry over to the stochastic one. However it is well known that the dynamics 
of stochastic systems can be very different from the dynamics of the corresponding deterministic systems, see for instance Mao (1997). Ideally we would like to deal directly with the dynamics of the stochastic systems, but this direct approach can be difficult.

A number of stochastic asset pricing models have been constructed in the heterogeneous agents literature. The earliest of which we are aware of is that of Föllmer (1974) who allows agents' preferences to be random and governed by a law that depends on their interaction with the economic environment. Rheinlaender and Steinkamp (2004) study a one-dimensional continuously randomised version of Zeeman's (1974) model and show a stochastic stabilisation effect and possible sudden trend reversal. Brock, Hommes and Wagener (2005) study the evolution of a discrete financial market model with many types of agents by focusing on the limiting distribution over types of agents. They show that the evolution can be well described by the large type limit (LTL) and that a simple version of LTL buffeted by noise is able to generate important stylised facts, such as volatility clustering and long memory, observed in real financial data. Föllmer, Horst and Kirman (2005) consider a discrete-time financial market model in which adaptive heterogeneous agents form their demands and switch among different expectations stochastically via a learning procedure. They show that, if the probability that an agent will switch to being a "chartist" is not too high, the limiting distribution of the price process exists, is unique and displays fat tails. Other related works include Hens and Schenk-Hoppé (2005) who analyse portfolio selection rules in incomplete markets where the wealth shares of investors are described by a discrete random dynamical system, Böhm and Chiarella (2005) who consider the dynamics of a general explicit random price process of many assets in an economy with overlapping generations of heterogeneous consumers forming optimal portfolios, Böhm and Wenzelburger (2005) who provide a simulation analysis of the empirical performance of portfolios in a competitive financial market with heterogeneous investors and show that the empirical performance measure may be misleading, and Horst and Wenzelburger (2007) who show how multiple stable long run distributions may emerge in a model with chartists. Most of the cited papers focus on the existence and uniqueness of limiting distributions of discrete time models. For continuous time models, we refer to the one-dimensional continuously randomised version of Zeeman's (1974) stock market model studied by Rheinlaender and Steinkamp (2004) and the work of Horst and Rothe (2008) who examine the impact of time lags in continuous time heterogeneous agent models.

In this paper, we extend the continuous-time deterministic models of speculative price dynamics of Beja and Goldman (1980) and Chiarella (1992) to a stochastic model, of which the market price equilibria can be characterised by stationary measures. We choose this very basic model of fundamentalist and speculative behaviour as it captures in a very simple way the essential aspects of the heterogeneous boundedly rational agents paradigm. By comparing both the indirect and direct approaches, we show that the stochastic model displays a bifurcation of very similar nature to that of the underlying deterministic model when the time lag is not too close to zero. Using the stochastic 
method of averaging, we show through a so-called phenomenological (P)-bifurcation analysis that the corresponding stationary measure displays a significant qualitative change near the threshold value from single-peak (unimodal) to crater-like (bimodal) joint distributions (and also marginal distributions) as the chartists become more active in the market. However, when the time lag in the formation of the price trends used by the chartists approaches zero, the stochastic model can display very different features from those of its underlying deterministic model. Economically, in the agent-based financial market model with stochastic noise, we study how the distributional properties of the model, which can be characterised by the stationary distribution of the market price process, change as agents' behaviour changes and how the market price distribution is influenced by the underlying deterministic dynamics. Mathematically, we seek to understand the connection between different types of attractors and bifurcations of the underlying deterministic skeleton and changes in stationary measures of the stochastic system. The issue of the consistency of the results under both the direct and indirect approaches is the main motivation of this paper.

The paper unfolds as follows. Section 2 reviews the heterogeneous agents financial market models developed by Beja and Goldman (1980) and Chiarella (1992). Sections 3 and 4 examine the dynamical behaviour of the stochastic model compared with that of the deterministic model when the time lag is positive or goes to zero, respectively. Section 5 concludes. All proofs are contained in the Appendix.

\section{The Model}

We consider a financial market consisting of investors with different beliefs. Based on the result of Boswijk, Hommes and Manzan (2007) (who estimate a heterogeneous agents model using S\&P500 market data) that the market mainly consists of fundamentalists and chartists, we assume that there are just two types of investors, fundamentalists and chartists, in the market. For simplicity, we also assume that there are only two types of assets, a risky asset (for instance a stock market index) and a riskless asset (typically a government bond). The fundamentalists base their investment decisions on an understanding of the fundamentals of the market, perhaps obtained through extensive statistical and economic analysis of various market factors. In contrast, the chartists do not necessarily have information about the fundamentals and their investment decisions are based on recent price trends. Following Beja and Goldman (1980) and Chiarella (1992), the changes of the risky asset price $P(t)$ are brought about by aggregate excess demand $D(t)$ of the fundamentalists $\left(D_{t}^{f}\right)$ and of the chartists $\left(D_{t}^{c}\right)$, defined below, at a finite speed of price adjustment. Furthermore in the market, the transactions and price adjustments are assumed to occur simultaneously. Accordingly, these assumptions may be expressed as

$$
d p(t)=D(t) d t=\left[D_{t}^{f}+D_{t}^{c}\right] d t,
$$


where $p_{t}=\ln P(t)$ is the logarithm of the risky asset price $P(t)$ at time $t$ and $D_{t}^{f}, D_{t}^{c}$ are the excess demands of the fundamentalists and chartists, respectively.

The excess demand of the fundamentalists is assumed to be given by

$$
D_{t}^{f}(p(t))=a[F(t)-p(t)]
$$

where $F(t)$ denotes the logarithm of the fundamental price ${ }^{1}$ that clears fundamental demand at time $t$ so that $D_{t}^{f}(F(t))=0$ and $a>0$ is a constant measuring the risk tolerance of the fundamentalists brought about by the market price deviation from the fundamental price. The linear excess demand function in (2.2) reflects the fundamentalists' superior knowledge on the fundamental price and strong belief in the convergence of the market price to the fundamental price at a speed measured by their risk tolerance.

Unlike the fundamentalists, the chartists do not necessarily know the fundamental price and they consider the opportunities afforded by the existence of continuous trading out of equilibrium. Their excess demand is assumed to reflect the potential for direct speculation on price changes, reflecting price momentum which is well documented in the literature, see for example Lee and Swaminathan (2000). Let $\psi(t)$ denote the chartists' assessment of the current trend in $p(t)$. Then the chartists' excess demand is assumed to be given by

$$
D_{t}^{c}(p(t))=h(\psi(t)),
$$

where $h$ is a nonlinear continuous and differentiable function, satisfying $h(0)=0, h^{\prime}(x)>$ 0 for $x \in \mathbb{R}, \lim _{x \rightarrow \pm \infty} h^{\prime}(x)=0 ; h^{\prime \prime}(x) x<0$ for $x \neq 0$ and $h^{(3)}(0)<0$ where $h^{(n)}$ denotes the $n$-th order derivative of $h(x)$ with respect to $x$. These properties imply that $h$ is an $S$-shaped function, indicating that when the expected trend in the price is above (below) zero, the chartists would like to hold a long (short) position in the risky asset. We also impose upper and lower bounds on the chartists' demand function, $\sup _{x}|h(x)|<\infty$, reflecting their budget constraints or cautiousness as the price trend becomes large in absolute value. The asymmetric treatment of the excess demands of the fundamentalists and chartists reflects their boundedly rational characteristics. With the knowledge of the fundamental price, the fundamentalists are confident about the mean-reversion of the market price to the fundamental price and are assumed to have no budget constraint. In contrast, the chartists do not necessarily have knowledge about the market fundamental price. They trade based on the market price trend and within their budget constraint. They extrapolate the market price when the trend is small, but become cautious when the trend is too strong. In addition, $h^{\prime}(\cdot)$ measures the intensity of the chartists' reaction to the long/short signal $\psi$. When $h^{\prime}(\cdot)$ is small, the chartists react weakly to the long/short signals as they believe it may have become unsustainably large in absolute value. Under the above assumptions, the intensity of the chartists' reaction is always bounded and in particular, the chartists are most sensitive at the equilibrium, that is $\max _{x} h^{\prime}(x)=h^{\prime}(0)$.

\footnotetext{
${ }^{1}$ We consider the market price and the fundamental price to both be detrended by the risk-free rate or correspondingly, the risk-free rate is assumed to be zero. Also, the fundamentalists are assumed to know the distribution of the fundamental price with infinite precision.
} 
Note that the chartists' speculation on the adjustment of the price primarily depends on an assessment of the state of the market as reflected in price trends. Typically, the assessment of the price trend is based at least in part on recent price changes and is an adaptive process of trend estimation. One of the simplest assumptions is that $\psi$ is taken as an exponentially declining weighted average of past price changes, that is, $\psi(t)=c \int_{-\infty}^{t} e^{-c(t-s)} d p(s)$, which can be expressed as the first order differential equation

$$
d \psi(t)=c[d p(t)-\psi(t) d t]
$$

where $c \in(0, \infty)$ is the decay rate, which can also be interpreted as the speed with which the chartists adjust their estimate of the trend to past price changes. Alternatively the quantity $\tau=1 / c$ may be viewed as the average time lag in the formation of expectations and it can be shown that ${ }^{2}$ in a loose sense $\psi(t) d t \approx d p(t-\tau)$.

Summarising the above set up, we obtain the asset price dynamics

$$
\left\{\begin{array}{l}
d p(t)=a[F(t)-p(t)] d t+h(\psi(t)) d t \\
d \psi(t)=\frac{1}{\tau}[-a p(t)-\psi(t)+h(\psi(t))+a F] d t
\end{array}\right.
$$

In the earlier cited literature that focuses on the deterministic dynamics of the financial market model, it is usually assumed that $F$ is constant. When the fundamental price $F$ follows a stochastic process, for example a random walk, (2.5) becomes a stochastic dynamical system. We shall examine the dynamical behaviour of (2.5) when the average time lag $\tau$ of the chartists in the formation of expectations is either positive or zero, the latter being treated as a limiting case of the former one as $\tau \rightarrow 0^{+}$.

\section{Dynamical Behaviour with Lagged Price Trend}

In this section, we first consider the dynamics of the deterministic model, followed by the dynamics of the stochastic model, in order to highlight the comparison between the deterministic and stochastic dynamics. Here we focus on $\tau$ strictly positive and discuss the limiting case $\tau \rightarrow 0^{+}$in Section 4 .

\subsection{The Deterministic Dynamical Behaviour}

When the $\log$ fundamental price is constant with $F \equiv F^{*}$, it is easy to verify that the corresponding deterministic model has a unique steady-state $\left(p^{*}, \psi^{*}\right)=\left(F^{*}, 0\right)$. Under the assumption that the chartists use a finite speed of adjustment in adapting to the price trend, that is $0<c<\infty$, or equivalently that the chartists always estimate the

\footnotetext{
${ }^{2}$ Equation $(2.4)$ can be written as $d p(t)=\tau d \psi(t)+\psi(t) d t \approx \psi(t+\tau) d t$. Hence $\psi(t) d t \approx d p(t-\tau)$. Alternatively one can calculate that the average time lag for the exponential weighting function is $\tau=1 / c$. So in an approximate sense $\psi$ is based on the change in $p$ evaluated at the average time lag $\tau$.
} 
price trend with a delay $\tau>0$, Beja and Goldman (1980) perform a local linear analysis around the steady-state. By considering the nonlinear nature of the function $h(x)$, Chiarella (1992) conducts a nonlinear analysis of the same model. Letting $b=h^{\prime}(0)$ represent the slope of the demand function at $\psi=0$ for the chartists, it is found that this parametner plays a very important role in determining the dynamics. In this paper, we take $b$ as the key parameter through which to examine the role of the chartists in determining the market price. The main result in Chiarella (1992) is summarised in the following theorem.

Theorem 3.1 If $F \equiv F^{*}$, the system (2.5) has a unique steady-state $\left(p^{*}, \psi^{*}\right)=\left(F^{*}, 0\right)$. Assume that $\tau>0$ and let $b=h^{\prime}(0)$ and $b^{*}=1+a \tau$, then $\left(p^{*}, \psi^{*}\right)$ is locally asymptotically stable for $b<b^{*}$ and unstable for $b>b^{*}$. In addition, $\left(p^{*}, \psi^{*}\right)$ undergoes a supercritical Hopf bifurcation at $b=b^{*}$ and a stable limit cycle exists for $b>b^{*}$.

To further study the impact of the behaviour of the fundamentalists and chartists on the price, we analyse the characteristics of the stable limit cycle generated from the supercritical Hopf bifurcation. We set $\phi=\dot{\psi}$ and then (2.5) can be transformed into the system

$$
\left\{\begin{array}{l}
\dot{\psi}=\phi \\
\dot{\phi}=\mathcal{K}(\psi, \phi)-\frac{a \psi}{\tau},
\end{array}\right.
$$

where $\mathcal{K}(\psi, \phi)=\left[\varsigma+h^{\prime}(\psi)-b\right] \phi / \tau$ and $\varsigma=b-b^{*}$. We consider $\psi$ in the neighborhood of 0 and restrict our analysis to be local. Making the polar coordinate transformation $\psi=\frac{r}{\eta} \sin (\theta-\eta t)$ and $\phi=r \cos (\theta-\eta t)$ where $\eta^{2}=a / \tau$, we can use the method of averaging (see Andronov, Vitt and Khaikin (1966)) to obtain a first order approximation to the amplitude function $r(t)$ satisfying

$$
\dot{r}=\mathscr{U}(r)=\frac{1}{2 \pi} \int_{0}^{2 \pi} \mathcal{K}\left(\frac{r}{\eta} \sin t, r \cos t\right) \cos t d t=\frac{r}{\tau}\left[H(r)-\frac{b}{2}+\frac{\varsigma}{2}\right],
$$

where $H(r)=\frac{1}{2 \pi} \int_{0}^{2 \pi} h^{\prime}\left(\frac{r}{\eta} \sin t\right) \cos ^{2} t d t$. The function $H(r)$ has the properties

$$
\begin{gathered}
H(0)=\frac{b}{2}, \lim _{r \rightarrow+\infty} H(r)=0 \text { and } H^{\prime}(r)<0 \text { for } r>0, \\
\mathbb{H}_{\max } \equiv \max _{r}\left\{H(r)-\frac{b}{2}+\frac{\varsigma}{2}\right\}=\frac{b-b^{*}}{2}, \mathbb{H}_{\text {min }} \equiv \min _{r}\left\{H(r)-\frac{b}{2}+\frac{\varsigma}{2}\right\}=\frac{-b^{*}}{2} .
\end{gathered}
$$

For the system (3.2), the steady state satisfies

$$
\mathscr{U}(r)=0
$$

When $b$ is small, in particular $b<b^{*}=1+a \tau$, then $\mathbb{H}_{\text {max }}<0$ and $r=0$ is a unique solution of (3.4). Thus $r=0$ is a unique steady state of (3.2) and stable. When $b$ is large, in particular $b>b^{*}=1+a \tau$, we have $\mathbb{H}_{\text {max }}>0$ and $\mathbb{H}_{\text {min }}<0$. Then (3.4) has 


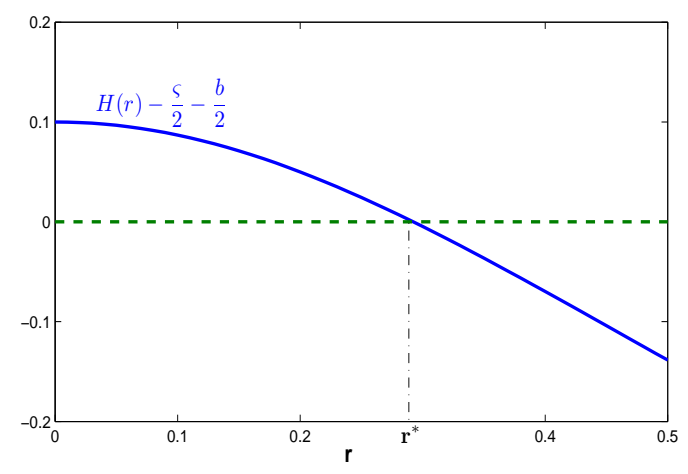

(a)

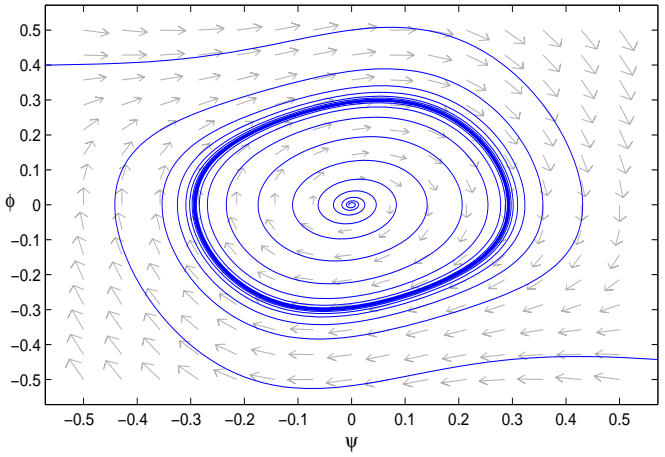

(b)

Figure 1: (a) The nonzero steady state of the polar radius in (3.2), and (b) the phase plot in $(\psi, \phi)$-plane. In both cases, $b>b^{*}$. We have taken $h(x)=\alpha \tanh (\beta x)$ with $a=1$, $\alpha=1, \beta=2.2$ and $\tau=1$ so that $b=\beta=2.2$ and $b^{*}=1+a \tau=2$.

a nonzero solution, denoted by $r^{*}$, see Fig. $1(\mathrm{a})^{3}$. From stability analysis, $r=r^{*}$ is a stable steady state of (3.2) while $r=0$ becomes an unstable steady state, as illustrated in Fig. 1(b).

By comparative static analysis, it is easy to see that the size of the limit cycle $r^{*}$ decreases with the increase of either the risk tolerance of the fundamentalists $(a)$ or the time lag used in the assessment of the current price trend by the chartists $(\tau)$. These results highlight the stabilising role of the fundamentalists and the destabilising influence of the chartists as they more actively adjust their estimate of the price trend.

\subsection{The Stochastic Dynamical Behaviour}

We now examine the stochastic dynamics generated from the randomness of the fundamental price. We assume that the log fundamental price $F(t)$ follows a random walk. That is, $F(t+h)-F(t)$ is normally distributed with mean 0 and variance $\sigma^{2} h$, independently of past values of $F(s)(s \leq t)$. Using the notation of the stochastic differential equation, the log fundamental value $F(t)$ can be considered to follow the Itô stochastic differential equation (SDE)

$$
d F=\sigma d W
$$

where $\sigma>0$ is the standard deviation (volatility) of the fundamental returns and $W$ is a standard Wiener process.

By incorporating the random log fundamental price process $F(t)$ of $(3.5)$ into the continuous-time model (2.5), we obtain the corresponding stochastic version of the financial market model. Setting $\phi d t=d \psi$, a nonlinear SDE system in $\psi$ and $\phi$ can be

\footnotetext{
${ }^{3}$ In this paper, we take $h(x)=\alpha \tanh (\beta x)$ in all numerical simulations.
} 
obtained, namely

$$
\left\{\begin{array}{l}
d \psi=\phi d t \\
d \phi=\frac{1}{\tau}\left[\left(\varsigma+h^{\prime}(\psi)-b\right) \phi d t-a \psi d t+a \sigma d W\right] .
\end{array}\right.
$$

Once the dynamics of $\psi(t)$ have been obtained, the dynamics of the price $p(t)$ can be obtained by integrating the first equation in (2.5).

Assume that $h^{\prime}(\psi)-b=h^{\prime}(\psi)-h^{\prime}(0)$ is small, that is $\psi$ is near 0. Following Arnold, Sri Namachchivaya and Schenk-Hoppé (1996), we restrict our analysis to be local and change parameters by rescaling (to "blow up" the neighbourhood around $\psi=0$ ) and introducing the (small) parameter $\varepsilon$ so that $h^{\prime}(\psi)-b \rightarrow \varepsilon^{2}\left(h^{\prime}(\psi)-b\right), \varsigma \rightarrow \varepsilon^{2} \varsigma, \sigma \rightarrow \varepsilon \sigma$. Then the SDE system (3.6) can be rewritten as

$$
\left\{\begin{array}{l}
d \psi=\phi d t \\
d \phi=\varepsilon^{2} \mathcal{K}(\psi, \phi) d t-\frac{a \psi}{\tau} d t+\frac{\varepsilon a \sigma}{\tau} d W
\end{array}\right.
$$

for which the corresponding Kolmogorov backward equation is

$$
\frac{\partial p_{\varepsilon}}{\partial t}=\phi \frac{\partial p_{\varepsilon}}{\partial \psi}-\eta^{2} \psi \frac{\partial p_{\varepsilon}}{\partial \phi}+\varepsilon^{2}\left[\frac{a^{2} \sigma^{2}}{\tau^{2}} \frac{\partial^{2} p_{\varepsilon}}{\partial \phi^{2}}+\mathcal{K}(\psi, \phi) \frac{\partial p_{\varepsilon}}{\partial \phi}\right]
$$

where $p_{\varepsilon}=p_{\varepsilon}\left(t ; \psi, \phi ; \psi_{1}, \phi_{1}\right)$ denotes the probability density of a transition from the point $(\psi, \phi)$ to the point $\left(\psi_{1}, \phi_{1}\right)$ in period of time $t$ for a trajectory of (3.7). We apply the same polar coordinate transformation $\psi=\frac{r}{\eta} \sin (\theta-\eta t), \phi=r \cos (\theta-\eta t)$ as in the deterministic case, and set

$$
\mathbb{u}_{\varepsilon}\left(t ; r, \theta ; r_{1}, \theta_{1}\right)=p_{\varepsilon}\left(t ; \frac{r}{\eta} \sin (\theta-\eta t), r \cos (\theta-\eta t) ; \frac{r_{1}}{\eta} \sin \left(\theta_{1}-\eta t\right), r_{1} \cos \left(\theta_{1}-\eta t\right)\right) .
$$

Then it can be verified that $\mathfrak{d}_{\varepsilon}$ satisfies

$$
\frac{\partial \mathbb{u}_{\varepsilon}}{\partial t}=\varepsilon^{2} L_{\varepsilon}(r, \theta, t) \mathfrak{u}_{\varepsilon},
$$

where the operator $L_{\varepsilon}(r, \theta, t)$ is given by

$$
\begin{aligned}
L_{\varepsilon}(r, \theta, t)= & \frac{a^{2} \sigma^{2}}{\tau^{2}}\left[\cos ^{2}(\theta-\eta t) \frac{\partial^{2}}{\partial r^{2}}-\frac{\sin 2(\theta-\eta t)}{r} \frac{\partial^{2}}{\partial r \partial \theta}+\frac{\sin ^{2}(\theta-\eta t)}{r^{2}} \frac{\partial^{2}}{\partial \theta^{2}}\right. \\
& \left.+\frac{\sin ^{2}(\theta-\eta t)}{r} \frac{\partial}{\partial r}+\frac{\sin 2(\theta-\eta t)}{r^{2}} \frac{\partial}{\partial \theta}\right] \\
& +\mathcal{K}\left(\frac{r}{\eta} \sin (\theta-\eta t), r \cos (\theta-\eta t)\right)\left[\cos (\theta-\eta t) \frac{\partial}{\partial r}-\frac{\sin (\theta-\eta t)}{r} \frac{\partial}{\partial \theta}\right],
\end{aligned}
$$

which is of the standard form to which the stochastic method of averaging can be applied. Using the techniques outlined in Khas'minskii (1963), the probability density of a 
transition of the random process $p_{0}\left(t ; r, \theta ; r_{1}, \theta_{1}\right)$ is found to satisfy the partial differential equation

$$
\frac{\partial p_{0}}{\partial t}=\frac{a^{2} \sigma^{2}}{2 \tau^{2}}\left[\frac{\partial^{2} p_{0}}{\partial r^{2}}+\frac{1}{r^{2}} \frac{\partial^{2} p_{0}}{\partial \theta^{2}}+\frac{1}{r} \frac{\partial p_{0}}{\partial r}\right]+\mathscr{U}(r) \frac{\partial p_{0}}{\partial r}-\frac{\mathscr{V}(r)}{r} \frac{\partial p_{0}}{\partial \theta}
$$

where $\mathscr{V}(r)=\frac{1}{2 \pi} \int_{0}^{2 \pi} \mathcal{K}\left(\frac{r}{\eta} \sin t, r \cos t\right) \sin t d t$. Then for any $R>0$ and $T>0$, it can be shown that

$$
\mathrm{u}_{\varepsilon}\left(t ; r, \theta ; r_{1}, \theta_{1}\right)-p_{0}\left(t \varepsilon^{2} ; r, \theta ; r_{1}, \theta_{1}\right) \rightarrow 0 \text { as } \varepsilon \rightarrow 0,
$$

uniformly with respect to $r, \theta, r_{1}, \theta_{1}$ for $r<R, r_{1}<R$ and with respect to $t$ for $0 \leq t \leq T / \varepsilon^{2}$. Making use of the fact that the stationary density of the two-dimensional process is $2 \pi$ periodic in $\theta$, we can assert that the stationary density $\mathrm{p}(r, \theta)$ corresponding to the solution of (3.9) is independent of $\theta$ and has the form $\mathbb{p}(r, \theta)=\frac{\mathbb{p}(r)}{2 \pi}$, where $\mathbb{p}(r)$ is the solution of the ordinary differential equation

$$
\frac{a^{2} \sigma^{2}}{2 \tau^{2}}\left[\frac{d^{2} \mathrm{p}(r)}{d r^{2}}-\frac{d}{d r} \frac{\mathrm{p}(r)}{r}\right]-\frac{d}{d r}(\mathscr{U}(r) \mathbb{p}(r))=0, \quad r \in[0,+\infty) .
$$

Furthermore, we can obtain the following result.

Theorem 3.2 There exists a unique stationary probability density of (3.10) given by

$$
\mathbb{p}(r)=C r \exp \left(\frac{2 \tau^{2}}{a^{2} \sigma^{2}} \int_{0}^{r} \mathscr{U}(s) d s\right)
$$

where $C$ is a normalisation constant.

Note that $\mathbb{p}(r)$ attains its extremum at the point $r=r_{e}$ satisfying

$$
\mathscr{U}\left(r_{e}\right)=-\frac{a^{2} \sigma^{2}}{2 \tau^{2} r_{e}}
$$

It is clear that when $\sigma=0,(3.11)$ reduces to the case of the steady state solution of the polar radius in the deterministic case, that is the one given by (3.4). Let $G(r)=-\frac{a^{2} \sigma^{2}}{2 \tau r^{2}}$ and then (3.11) may be written as

$$
H(r)+\frac{\varsigma}{2}-\frac{b}{2}=G(r) .
$$

By (3.3) and the monotonicity of $G(\cdot)$ for $r \in(0, \infty),(3.12)$ only has one solution $r=r_{e}$ and in particular, $\mathrm{p}(\cdot)$ attains its maximum value at $r=r_{e}$.

When $b$ is small, in particular $b<b^{*}=1+a \tau$, then $\mathbb{H}_{\max }<0$ and (3.12) is approximated by $-\frac{a^{2} \sigma^{2}}{2 \tau r^{2}}=\frac{\varsigma}{2}$. Then $\mathrm{p}(r)$ attains its maximum value near $r_{e} \approx \frac{a \sigma}{\sqrt{-\varsigma \tau}}$, which is close to zero for small $\sigma$, see Fig. 2(a). This in turn implies a joint stationary density of $(\psi, \phi)$ with a single peak around the origin, as illustrated in Fig. $3(\mathrm{a})^{4}$. In

\footnotetext{
${ }^{4}$ Note that the distributions displayed in Fig. 3 are those of the system (3.6) and the first equation of (2.5), obtained by use of the Euler-Maruyama scheme with one sample path up to time 500,000.
} 


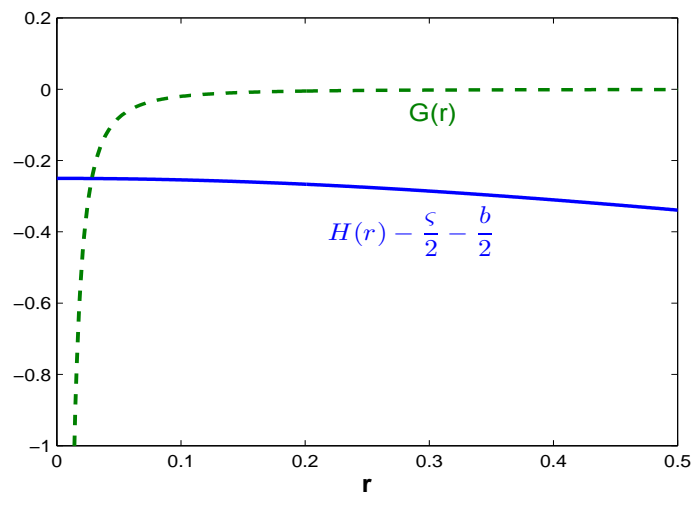

(a) $b=1.5<b^{*}$

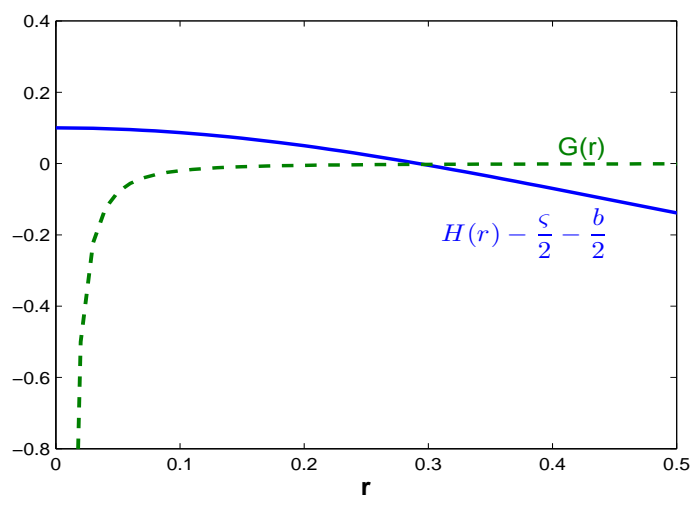

(b) $b=2.2>b^{*}$

Figure 2: The functions $G$ and $H$ and the determination of $r_{e}$ (a) for $b<b^{*}$ and (b) for $b>b^{*}$. Here $a=1, \sigma=0.02$ and $h(x)=\alpha \tanh (\beta x)$ with $\alpha=1$ so that $b=\beta$ and $b^{*}=1+a \tau=2$.

particular, when $\sigma \rightarrow 0$, we have $r_{e} \rightarrow 0$ consistently with the deterministic case. When $b$ is large, in particular $b>b^{*}=1+a \tau$, we have $\mathbb{H}_{\text {max }}>0$ and $\mathbb{H}_{\text {min }}<0$. In this case, the solution of (3.12) is far away from zero, as shown in Fig. 2(b) which can be regarded as the stochastic version of Fig. 1(a). This indicates a crater-like density whose maximum is located on a circle (around $r=0$ which is the steady state of the deterministic system) with a large radius, shown in Fig. 3(g). Figure 3(d) illustrates the distribution of the transition from the single-peak to the crater-like density distribution. The plots in the second and third columns of Fig. 3 illustrate the corresponding marginal distributions of $\psi$ and $p$.

From the above analysis, we see the qualitative change of the stationary density when the parameter $b$ changes. If we treat this stationary density (when $b$ is large) as a bifurcation from the case when $b$ is small, the maximum radius of the density function corresponds to a Hopf bifurcation. In stochastic bifurcation theory, such a bifurcation is known as a phenomenological $(P)$-bifurcation ${ }^{5}$. It is in this sense that we argue that the stochastic model shares the corresponding dynamics to those of the deterministic model.

\section{Dynamical Behaviour in the Limit $\tau \rightarrow 0^{+}$}

In this section, we consider the limiting case $\tau \rightarrow 0^{+}$. This corresponds to the situation in which the chartists use the most recent price change to estimate the trend of the price. Different from the previous case $\tau>0$, the limiting case is of interest because it has a similar structure to the catastrophe theory model of Zeeman (1974), a structure

\footnotetext{
${ }^{5}$ The P-bifurcation approach to stochastic bifurcation theory is used to examine the qualitative changes of the stationary measure when a parameter varies, which is based on the definition given by Arnold (1998).
} 


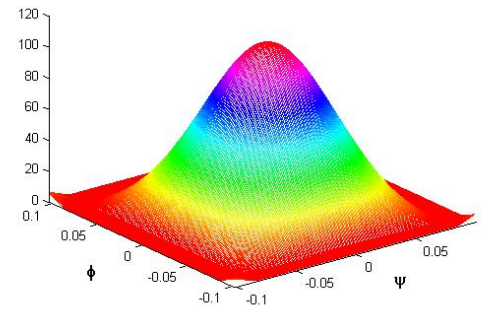

(a) joint distribution with $b=1.5$

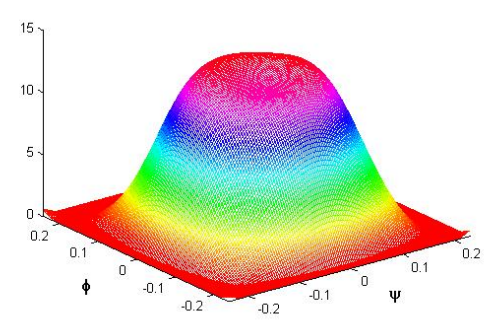

(d) joint distribution with $b=2.0$

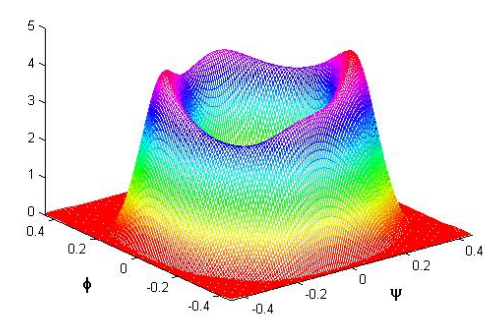

(g) joint distribution with $b=2.2$

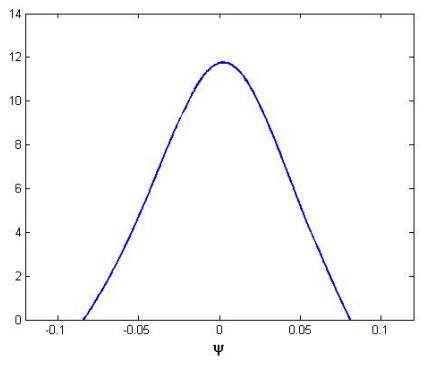

(b) marginal distribution of $\psi$

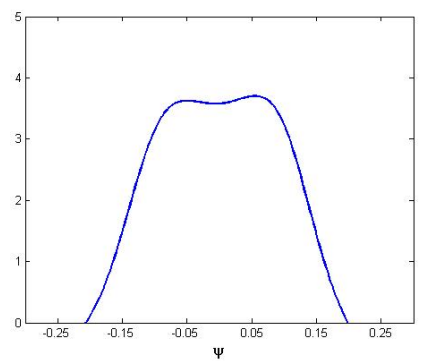

(e) marginal distribution of $\psi$

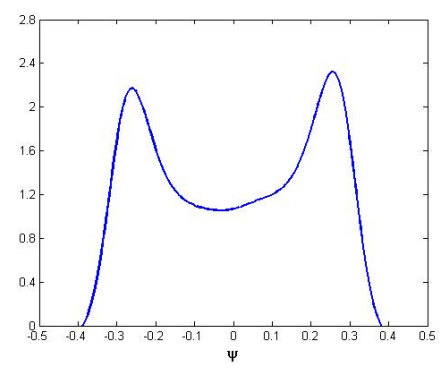

(h) marginal distribution of $\psi$

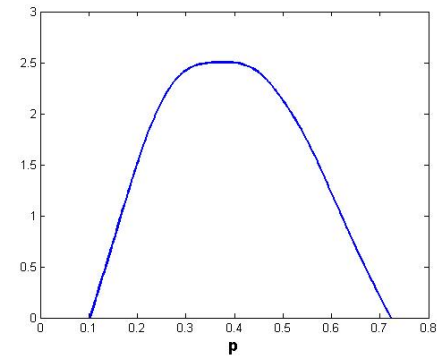

(c) marginal distribution of $p$

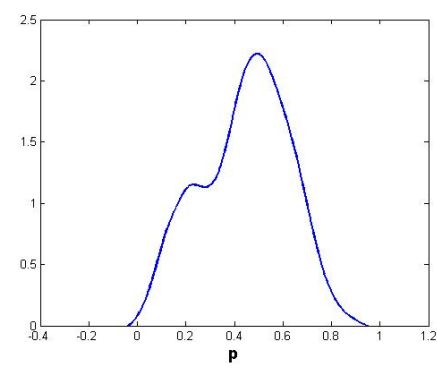

(f) marginal distribution of $p$

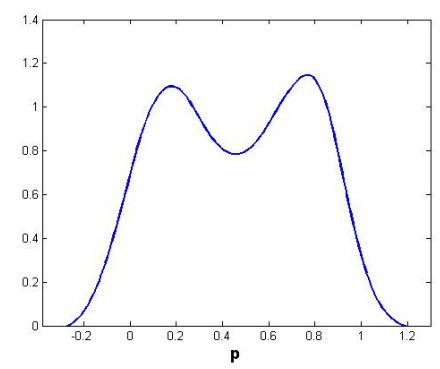

(i) marginal distribution of $p$

Figure 3: Joint stationary densities of $\psi$ and $\phi$ and the corresponding marginal distributions for $\psi$ and $p$. We have taken $h(x)=\alpha \tanh (\beta x)$ with $a=1, \alpha=1, \tau=1, \sigma=0.02$, so that $b=\beta$ and $b^{*}=1+a \tau=2$.

that has been suggested by some empirical studies, such as Anderson (1989). Similar to the approach of Section 3, we first consider the deterministic model and then move to the stochastic model.

\subsection{The Deterministic Dynamical Behaviour}

When $F \equiv F^{*}$, the system $(2.5)$ can be rewritten as

$$
\Sigma_{\tau}:\left\{\begin{array}{l}
\dot{p}=f(p, \psi):=a\left(F^{*}-p\right)+h(\psi) \\
\tau \dot{\psi}=g(p, \psi):=a\left(F^{*}-p\right)+h(\psi)-\psi
\end{array}\right.
$$

When $\tau \rightarrow 0^{+}$, dynamical systems such as (4.1) are known as singularly perturbed systems. As we show in the following, the dynamics have the catastrophe theory characteristics, that is the dynamics are fast in one direction (here the $\psi$-direction) and slow 
in the other direction (here the $p$-direction), see Yurkevich (2004) for a more detailed study of such systems.

In fact, in the limiting case $\tau \rightarrow 0^{+}$, the system (4.1) becomes

$$
\Sigma_{0}:\left\{\begin{array}{l}
\dot{p}=f(p, \psi), \\
0=g(p, \psi),
\end{array}\right.
$$

which is a differential-algebraic equation $(\mathrm{DAE})^{6}$ with an algebraic constraint on the variables. The dynamical system $\Sigma_{\tau}$ has apparently lost a dimension in the limit $\tau=0$. By differentiating the second equation of $\Sigma_{0}$ with respect to $t$ and using the fact that $\dot{p}=\psi$, we obtain the result that the dynamics of $\psi$ are given by

$$
\left(1-h^{\prime}(\psi)\right) \dot{\psi}=-a \psi
$$

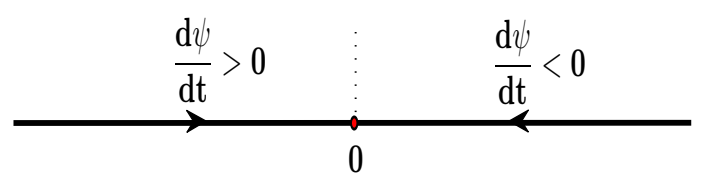

(a) $b<1$ : the unique steady state is stable.

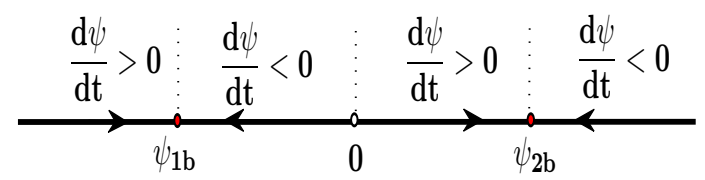

(b) $b>1$ and $h^{\prime}\left(\psi_{1 b}\right)=h^{\prime}\left(\psi_{2 b}\right)=1$ : one steady state and two singular points.

Figure 4: The vector field of $\psi$.

Note that when $b<1, h^{\prime}(\psi)<1$ for all $\psi$, so that in this case $\psi=0$ is a unique steady state of (4.3) and is stable, as illustrated in Fig. 4(a). However, when $b>1$, there exist two values $\psi_{1 b}$ and $\psi_{2 b}$ with $\psi_{1 b}<0<\psi_{2 b}$ such that $h^{\prime}\left(\psi_{1 b}\right)=h^{\prime}\left(\psi_{2 b}\right)=1$. These values are shown in Fig. 4(b) along with the signs of $\dot{\psi}$, obtained from (4.3), at various values of $\psi$. By the analysis of the vector field of $\psi$, the flow of $\psi$ with nonzero initial value moves away from the origin towards $\psi_{1 b}$ or $\psi_{2 b}$. This means that the steady state $\psi=0$ loses its stability at $b=1$ and becomes unstable as $b$ increases from $1^{-}$to $1^{+}$. In addition, note that when there is a value $\psi_{*}$ such that $h^{\prime}\left(\psi_{*}\right)=1$, then the solution of the second equation of $\Sigma_{0}$ with respect to $\psi$ is nonunique because the Implicit Function Theorem does not hold at $\psi=\psi_{*}$, which is known as a singular point. At the singular point, $\left.\dot{\psi}\right|_{\psi=\psi_{*}}=\infty$. Thus, singular phenomena occur for $b \geq 1$.

For system $\Sigma_{0}$, its singularity, the bifurcation of the fundamental equilibrium $(p, \psi)=$ $\left(F^{*}, 0\right)$ near the singular parameter (the so-called singularity induced bifurcation), and its dynamics are described by the following theorem ${ }^{7}$.

Theorem 4.1 A singularity induced bifurcation occurs at $b=1$ and the steady state $\left(F^{*}, 0\right)$ loses its stability at $b=1$ and becomes unstable as $b$ increases from $1^{-}$to $1^{+}$.

\footnotetext{
${ }^{6}$ For more information about DAEs, we refer the reader to the book of Brenan, Campbell and Petzold (1989).

${ }^{7}$ For the more information about the proof of singularity induced bifurcations, we refer the reader to Chiarella, He and Zheng (2008).
} 


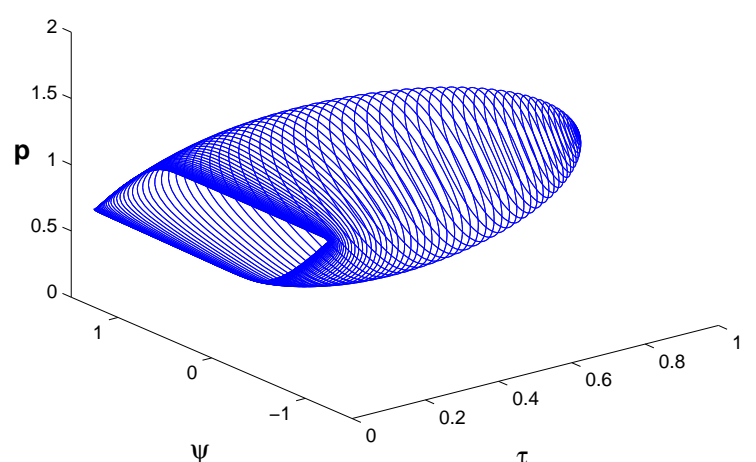

(a) The limit cycle persists when $\tau$ decreases to zero

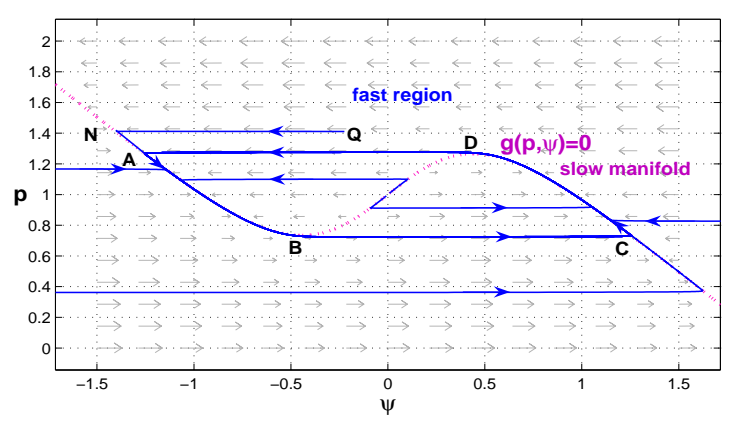

(c) Jump phenomenon as $\tau \rightarrow 0^{+}$

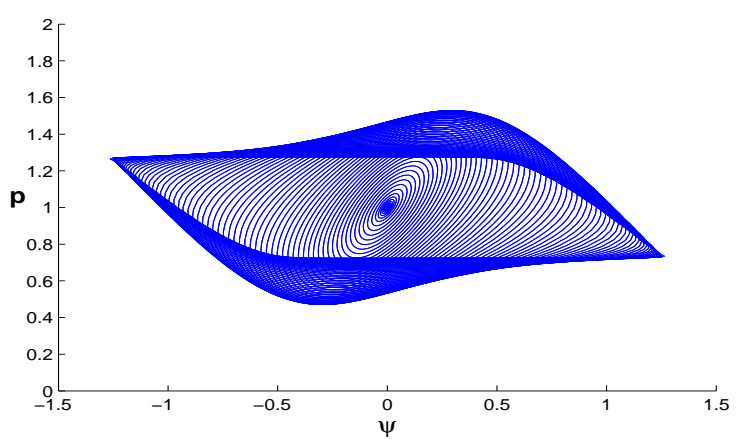

(b) The projection of (a)
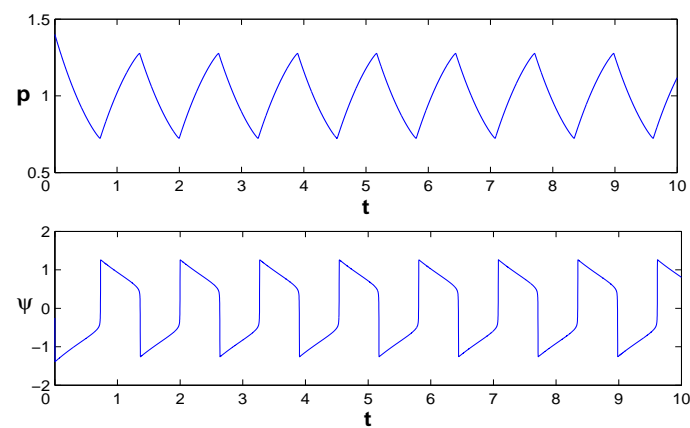

(d) Time series as $\tau \rightarrow 0^{+}$

Figure 5: (a) The limiting limit cycle as $\tau \rightarrow 0^{+}$; (b) the projection of (a) onto the $(\psi, p)$-plane with the parallelogram-like figure being the limiting case $\tau \rightarrow 0^{+}$; (c) the jump fluctuation in the phase plane and $(\mathrm{d})$ the corresponding time series of $p(t)$ and $\psi(t)$ at $\tau \rightarrow 0^{+}$. Here $a=1$ and $h(x)=\alpha \tanh (\beta x)$ with $\alpha=1$ and $\beta=2.2$.

To understand the complete dynamics of the system $\Sigma_{0}$, we consider the limiting dynamics of the singularly perturbed system $\Sigma_{\tau}$ as $\tau \rightarrow 0^{+}$. We shall show that the singular phenomenon for the system $\Sigma_{0}$ corresponds to the limiting case of the Hopf Bifurcation in the system $\Sigma_{\tau}$ for $b^{*}=1+a \tau(\tau \neq 0)$ when $\tau \rightarrow 0^{+}$.

Recall that, at $b^{*}=b^{*}(\tau)=1+\tau a$, the system $\Sigma_{\tau}$ has a pair of purely imaginary eigenvalues $\lambda_{1,2}= \pm \sqrt{-a / \tau}$ and when $\tau \rightarrow 0^{+}, b^{*} \rightarrow 1$ and $\left|\lambda_{1,2}\right| \rightarrow \infty$. With a fixed $b>b^{*}\left(\tau_{0}\right)\left(\tau_{0}>0\right)$, as $\tau \rightarrow 0^{+}$, the limit cycle persists, as suggested by the numerical simulations in Fig. 5(a). Figure 5(b) gives a projection of Fig. 5(a) onto the $(\psi, p)$-plane. In fact, in the $(\psi, p)$-plane, the following observations are made about the system $\Sigma_{0}$. As illustrated in Fig. $5(\mathrm{c})$, for $g(p, \psi) \neq 0, \psi$ moves infinitely rapidly toward the curve defined by $g(p, \psi)=0$ since $\dot{\psi} \rightarrow \infty$ as $\tau \rightarrow 0^{+}$, such a region is denoted as the fast region. For $g(p, \psi)=0$, as $\tau \rightarrow 0^{+}$the dynamics are governed by the differential equation for $p$, namely $\dot{p}=a\left(F^{*}-p\right)+h(\psi)$, along the curve $g(p, \psi)=0$ which is called the slow manifold. Specifically, consider how the motion evolves from the initial point $Q$ in the fast region in Fig. 5(c). The variable $\psi$ moves instantaneously 
horizontally to the point $N$ on the slow manifold $g(p, \psi)=0$. Motion is then down the slow manifold under the influence of $\dot{p}=a\left(F^{*}-p\right)+h(\psi)$. When the singular point $B$ is reached, which corresponds to $\psi_{1 b}$ in Fig. 4(b), $\psi$ jumps instantaneously horizontally across to $C$ on the opposite branch of the slow manifold. Motion is then up to another singular point $D$ which corresponds to $\psi_{2 b}$ in Fig. 4 (b) and the cycle then repeats itself. Therefore, the singular phenomenon in system $\Sigma_{0}$ corresponds to a jump phenomenon and the limit cycle with the jump phenomenon consists of two slow movements along the manifold of $\Sigma_{0}$ shown in Fig. $5(\mathrm{c}), A \rightarrow B$, and $C \rightarrow D$, and two jumps at the singular points $B$ and $D$ in $\Sigma_{0}$, namely $B \rightarrow C$ and $D \rightarrow A$. The corresponding time series in Fig. $5(\mathrm{~d})$ clearly shows the periodic slow movement in price $p$ and sudden jumps in $\psi$ from time to time. This phenomenon indicates that the model is able to generate significant transitory and predictable fluctuations around the equilibrium. Using a different approach, this jump fluctuation phenomenon in the model of fundamentalists and chartists was studied by Chiarella (1992) who points out that it is merely the relaxation oscillation well-known in mechanics and expounded for example by Grasman (1987). Our analysis shows that strong reaction to price changes by the chartists can make the fundamental price unstable, leading to predictable cycles for the market prices and jumps in their estimate of the price trends.

\subsection{The Stochastic Dynamical Behaviour}

We now analyse the behaviour of the stochastic dynamics in the limit $\tau \rightarrow 0^{+}$. As $\tau \rightarrow 0^{+}$, we see from (2.4) that $d p \rightarrow \psi d t$ whilst from the first equation of $(2.5)$, the log price $p$ is governed by $d p=[a(F-p)+h(\psi)] d t$. It follows that $\psi=a(F-p)+h(\psi)$, which implies that $\psi$ is a continuous process. Assuming that $\psi$ is an Itô process of the form

$$
d \psi=\mathbb{M} d t+\sigma d W
$$

and taking the stochastic differential of the two sides of $\psi=a(F-p)+h(\psi)$, we find that

$$
\begin{aligned}
d \psi & =a(d F-d p)+h^{\prime}(\psi) d \psi+\frac{1}{2} h^{\prime \prime}(\psi)(d \psi)^{2} \\
& =a(\sigma d W-\psi d t)+h^{\prime}(\psi)(\mathbb{M} d t+\sigma d W)+\frac{1}{2} h^{\prime \prime}(\psi) \sigma^{2} d t
\end{aligned}
$$

from which after some algebraic manipulations and comparing with (4.4), we obtain the result that

$$
\sigma=\sigma(\psi)=\frac{a \sigma}{1-h^{\prime}(\psi)} \quad \text { and } \quad \mathbb{M}=\mathbb{M}(\psi)=\frac{-a \psi}{1-h^{\prime}(\psi)}+\frac{1}{2} \sigma^{\prime}(\psi) \sigma(\psi)
$$

Hence, if there exists $\psi_{*}$ such that $h^{\prime}\left(\psi_{*}\right)=1$, then (4.4) is singular at $\psi=\psi_{*}$. Similarly to the deterministic case, for the different cases, $b<1, b=1$ and $b>1$, the stochastic differential equation (4.4) will have a different number of singular points and therefore 
exhibit different behaviour. We will now discuss each case in turn. To simplify the analysis, in this subsection, we make some technical assumptions that there exists $x_{1}<$ $0<x_{2}$ such that $h^{(3)}(\psi)<0$ for $\psi \in\left(x_{1}, x_{2}\right), h^{(3)}(\psi)>0$ otherwise, and $\psi h^{(4)}(\psi)>0$ for $\psi \in\left(x_{1}, x_{2}\right)$, as illustrated in Fig. 6 . These conditions are satisfied by the hyperbolic tangent function used in the numerical simulations of this paper.

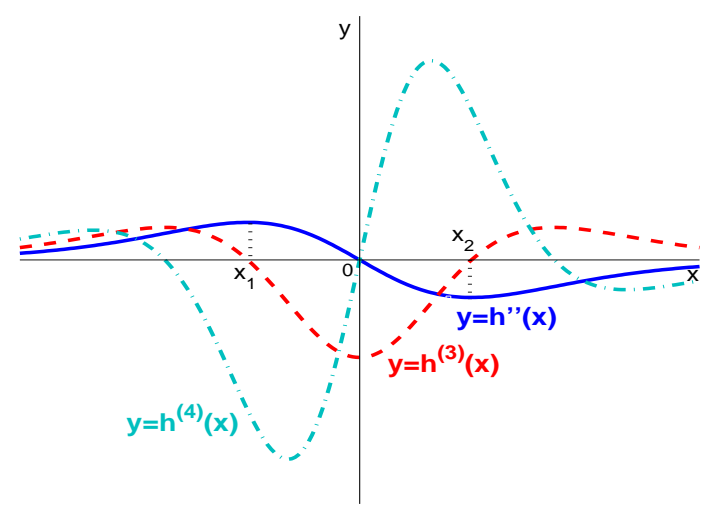

Figure 6: Plots of functions $h^{\prime \prime}(x), h^{(3)}(x)$ and $h^{(4)}(x)$.

When $b<1$, we have $1-h^{\prime}(\psi)>0$ for any $\psi$ and there is no singularity in $(-\infty,+\infty)$. The only singular points are $\pm \infty$. Based on the theory of the classification of singular boundaries $^{8}$, we obtain the following result.

Theorem 4.2 When $b<1$, there exists a unique stationary density $\mathbb{p}$ for $\psi$, given by

$$
\mathbb{p}(\psi)=N \frac{\left(1-h^{\prime}(\psi)\right)}{a \sigma} \exp \left(\int_{0}^{\psi}-\frac{2 y\left(1-h^{\prime}(y)\right)}{a \sigma^{2}} d y\right)
$$

and $N$ is a normalisation constant.

Note that when $\psi$ satisfies

$$
h^{\prime \prime}(\psi)+\frac{2 \psi\left(1-h^{\prime}(\psi)\right)^{2}}{a \sigma^{2}}=0
$$

the stationary density $\mathbb{p}(\cdot)$ attains its extremum. This, together with the assumptions on the function $h$, leads to the following result on the P-bifurcation with respect to $\psi$.

Theorem 4.3 (P-Bifurcation) Let $b_{p}=1-\sqrt{-\frac{h^{(3)}(0) a \sigma^{2}}{2}}$.

(1) When $h^{(3)}(0)>-\frac{2}{a \sigma^{2}}$ and $0<b<b_{p}$, the stationary density $\mathbb{p}(\cdot)$ has a unique extreme point $\psi=0$, at which $\mathbb{p}(\cdot)$ attains its maximum.

\footnotetext{
${ }^{8}$ We refer to Lin and Cai (2004) for more information about the theory of the classification of various singular boundaries, including entrance, regular, and (attractively and repulsively) natural boundaries used in our discussion.
} 


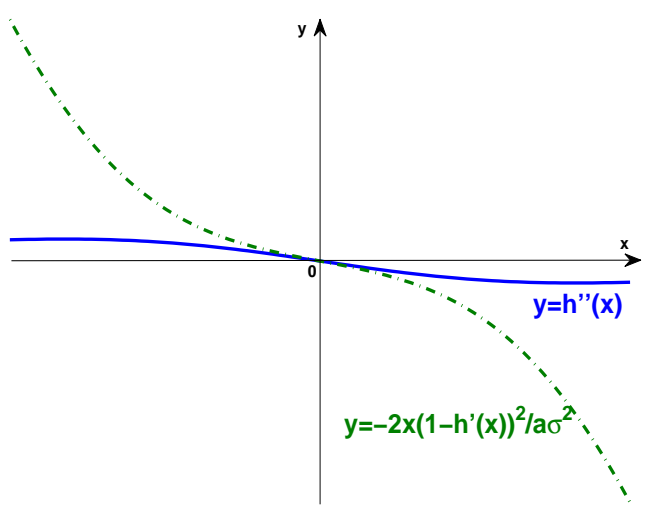

(a) $h^{(3)}(0)>-\frac{2}{a \sigma^{2}}$ and $b<b_{p}$

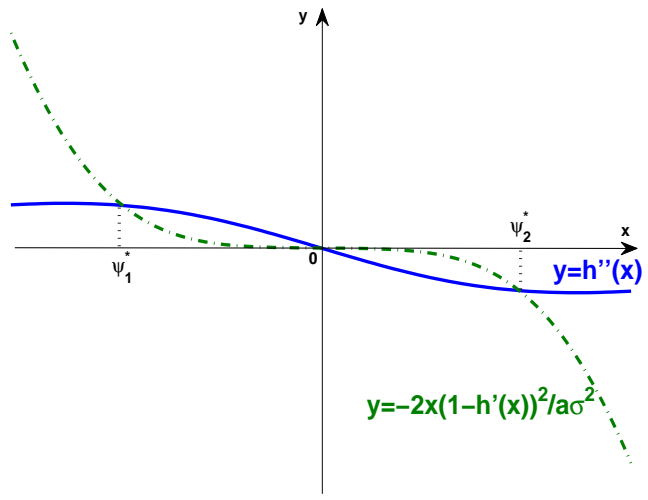

(b) $\max \left\{b_{p}, 0\right\}<b<1$

Figure 7: The determination of the extreme points of the stationary density $\mathbb{p}(\cdot)$

(2) When $\max \left\{b_{p}, 0\right\}<b<1$, the stationary density $\mathbb{p}(\cdot)$ has three extreme points $\psi_{1}^{*}$, 0 and $\psi_{2}^{*}$ satisfying $\psi_{1}^{*}<0<\psi_{2}^{*}$. In addition, the stationary density $\mathbb{p}(\cdot)$ attains its minimum value at $\psi=0$ and its maximum values at $\psi=\psi_{1}^{*}$ and $\psi_{2}^{*}$.

Theorem 4.3 indicates that, as the chartists place more and more weight on the recent price changes (that is $\tau \rightarrow 0^{+}$), the number of the extreme points of the stationary density $\mathbb{p}(\cdot)$ in (4.6) changes from one to three as the parameter $b$ changes (but $b<1$ ). This is shown in Fig. 7. This means that, a moderate increase in activity (such that $b<1$ ) of the chartists who weight the recent price changes very heavily results in a large deviation of their estimate of the price trend $\psi$ from its mean value, illustrated by the changes ${ }^{9}$ from the unimodal to bimodal stationary distribution in the upper panel of Fig. 8. The changes are further illustrated by the underlying time series for $\psi$ in the bottom panel of Fig. 8.

The appearance of the P-bifurcation discussed above cannot be inferred from any information in the corresponding deterministic case because when $b<1$, Theorem 4.1 implies that in the deterministic model, the unique steady state $\left(F^{*}, 0\right)$ is always stable and there is no bifurcation ${ }^{10}$. This result illustrates that, from the P-bifurcation point of

\footnotetext{
${ }^{9}$ In Figs 8 and 9, we have taken $h(x)=\alpha \tanh (\beta x)$ with $\beta=1, a=1$ and $\sigma=0.1$ so that $b=\alpha$. In Fig. $8, \alpha=0.7, \alpha=0.9$ and $\alpha=0.95$ for the first, second and third columns and in Fig. $9, \alpha=1.2$ so that $b=\alpha>1$.

${ }^{10}$ The phenomenological (P)-bifurcation analysis focuses on the stationary distribution and is, in general, not related to path-wise stability. There is another type of stochastic bifurcation, known as the dynamical (D)-bifurcation approach which does focus on path-wise stability and is based on the invariant measure and the multiplicative ergodic theorem. By using D-bifurcation analysis, Chiarella, He, Wang and Zheng (2008) (see also Chiarella, He and Zheng (2008) for complete details) show that the random dynamical system generated from (4.4) has no D-bifurcation since the invariant measure about $\psi$ is unique and always has a negative Lyapunov exponent. This result is consistent with the stable case in the corresponding deterministic model when $b<1$.
} 


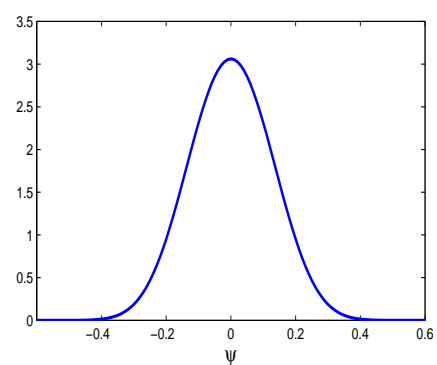

(a) Density of $\psi$ for $b<b_{p}$

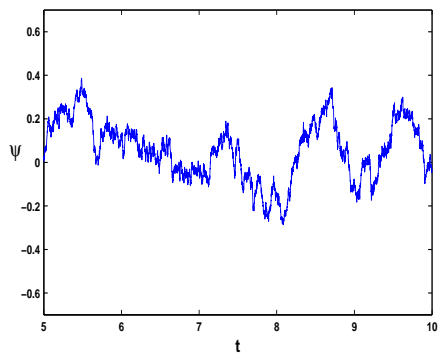

(d) Time series of $\psi$ for $b<b_{p}$

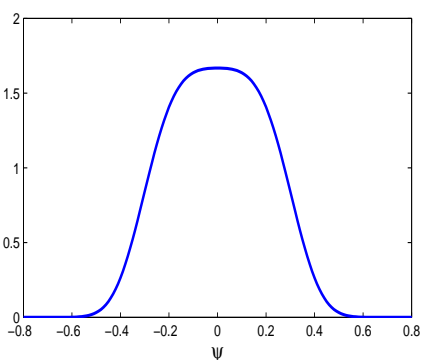

(b) Density of $\psi$ for $b \lesssim b_{p}$

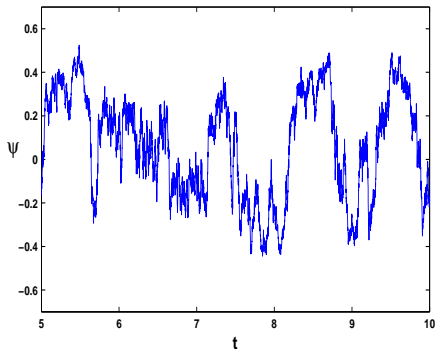

(e) Time series of $\psi$ for $b \lesssim b_{p}$

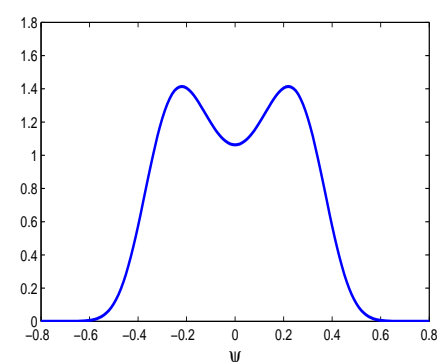

(c) Density of $\psi$ for $b_{p} \lesssim b<1$

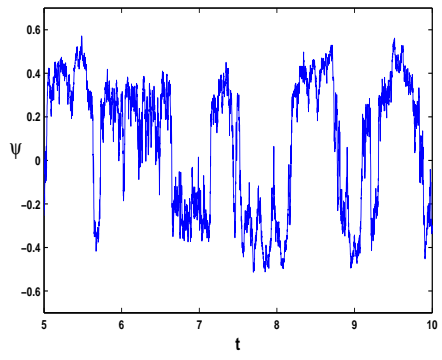

(f) Time series of $\psi$ for $b_{p} \lesssim b<1$

Figure 8: When $\tau \rightarrow 0^{+}$and $h^{(3)}(0)>-\frac{2}{a \sigma^{2}}$, a $P$-bifurcation occurs at $b=b_{p}$. The top panel shows different typical stationary densities $\mathbb{p}$ of $\psi$ with different parameter ranges and the bottom panel shows the underlying time series.

view, the stochastic dynamical system can be very different from that of the underlying deterministic system ${ }^{11}$.

When $b=1$, that is $h^{\prime}(0)=1$, the drift term $\mathbb{M}(0)$ in (4.4) becomes unbounded and furthermore $\psi=0$ is a regular boundary. With the increase of $b$ to $b>1$, there exist $\psi_{1 b}<0<\psi_{2 b}$ satisfying $h^{\prime}\left(\psi_{1 b}\right)=h^{\prime}\left(\psi_{2 b}\right)=1$, both of which are also regular boundaries. The appearance of the regular boundary at $b=1$ corresponds to the occurrence of a singularity induced bifurcation in the corresponding deterministic case and $\psi_{1 b}, \psi_{2 b}$ correspond to the jump points in the deterministic case. In fact, at the same time, the stochastic model may share some of the features of the deterministic model. For example, when $b>1$, we know from the previous section that the deterministic dynamics exhibit fast motion in the $\psi$-direction and slow motion in the $p$-direction. For the stochastic model, we observe a similar behaviour, as suggested by the simulations in Fig. 9. However, once a regular boundary appears, it renders the stationary solution

\footnotetext{
${ }^{11}$ This observed difference is based on Arnold's definition of the P-bifurcation. The qualitative change of the stationary distributions under change of variables is also discussed in Wagenmarkers, Molenarra, Grasman, Hartelman and van der Maas (2005) for continuous time random dynamical systems (see also Diks and Wagener (2006) for discrete time random dynamical systems), in a way different from that of Arnold, these authors use the level-crossing function to characterise the change. Correspondingly, this difference between the deterministic and stochastic cases in the paper observed under Arnold's definition may disappear, but this issue is beyond the scope of our discussion.
} 

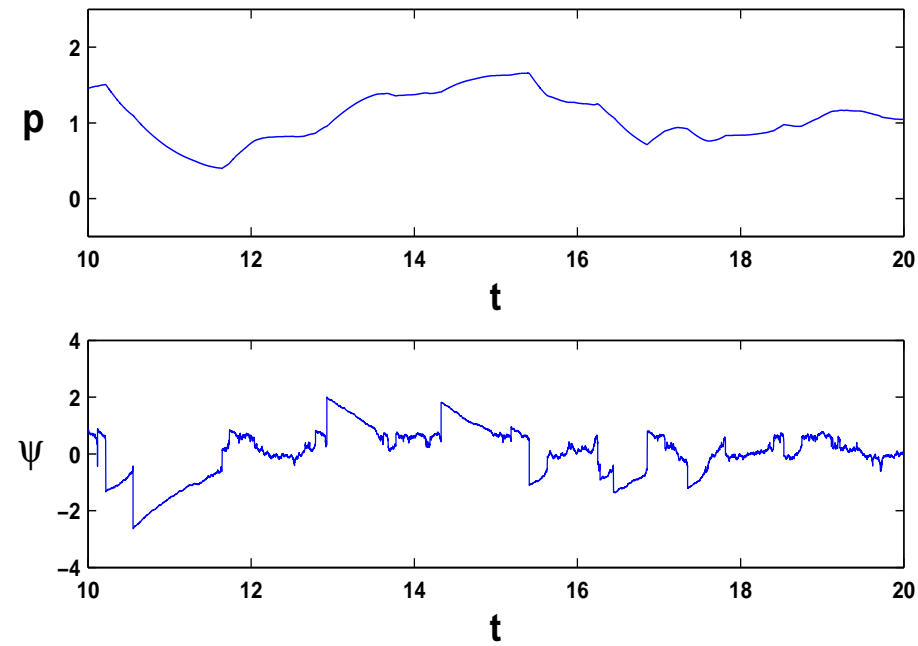

Figure 9: Fast motion in $\psi$ and slow motion in $p$ in the limit of $\tau \rightarrow 0^{+}$for $b>1$

nonunique $^{12}$ without further stipulation on the behaviour of (4.4) (see Feller (1952)). Unlike the case of $b<1$, this causes some difficulties in obtaining analytical properties of the stationary densities of the stochastic system. However, from a practical point of view, this additional freedom may be a blessing since it may permit certain realistic behaviour to be incorporated into the context of the financial model, but we leave such considerations to future research.

\section{Conclusion}

In this paper, within the framework of the heterogeneous agent paradigm, we extend the basic deterministic model of Beja and Goldman (1980) and Chiarella (1992) to a stochastic model for the market price, and examine the consistency of the stochastic dynamics under the indirect and direct approaches. By using $\mathrm{P}$ (Phenomenological)bifurcation analysis, we examine the qualitative changes of the stationary measures of the stochastic model.

For the simple stochastic financial market model studied here, when the time lag used by the chartists to form their expected price trends is not zero, we show that near the steady state of the underlying deterministic model, the approximation of the stochastic model shares the corresponding Hopf bifurcation dynamics of the deterministic model. When the time lag used by the chartists to form their expected price changes approaches zero, so that the chartists are placing more and more weight on recent price changes, then the system can have singular points. In this case, the stochastic model displays very different dynamics from those of the underlying deterministic model. In particular,

\footnotetext{
${ }^{12}$ Note that in the deterministic case, the occurrence of the singularity induced bifurcation corresponds to the appearance of a singular point where the Implicit Function Theorem does not hold, hence the solution for the implicit function can be nonunique.
} 
the fundamental noise can destabilise the market equilibrium and result in a change of the stationary distribution through a P-bifurcation of the stochastic model, while the corresponding deterministic model displays no bifurcation. Our results demonstrate the important connection, but also the significant difference, of the dynamics between deterministic and stochastic models.

Economically, we have considered a very simple financial market model with heterogeneous agents. This stylised model and the role played by the chartists are shared by many heterogeneous agent-based discrete-time models in recent literature. However rigorous analysis and theoretical understanding of the stochastic behaviour underlying this intuition are important to policy analysis, investment strategies and market design, but have drawn less attention, mainly due to the difficult nature of these issues. Ultimately we need to apply the theory of stochastic bifurcation directly rather than simulate the models indirectly. Applications of the tools and concepts of stochastic bifurcation theory to financial market models to obtain analytic insight into these intuitions and to improve our understanding about the interaction of the behaviour of heterogeneous agents and noise are the main contributions of this paper.

In order to bring out the basic phenomena associated with stochastic bifurcation we have focused on a highly simplified model. The simplicity of the model limits its ability to generate the stylised facts observed in financial markets. In order to do so the model would need to be embellished in a number of ways in future research, in particular: deriving the asset demands of agents from an intertemporal optimisation framework; introducing other types of randomness such as market noise to characterise the excess volatility; allowing for switching of strategies according to some fitness measure (see for example Brock and Hommes (1997, 1998)); and analysing in closer detail the stochastic dynamics to replicate the stylised facts and power-law behaviour.

\section{Appendix}

\section{A1. Proof of Theorem 3.2}

The proof is based on the theory of the classification of singular boundaries in Lin and Cai (2004). First, note that (3.10) has two boundaries $r_{l}=0$ and $r_{r}=+\infty$. The drift and diffusion coefficients corresponding to (3.10) are respectively $\mathbf{M}(r)=\mathscr{U}(r)+\frac{a^{2} \sigma^{2}}{2 \tau^{2} r}$ and $\varrho^{2}(r)=\frac{a^{2} \sigma^{2}}{\tau^{2}}$. It is easy to see that the left boundary $r_{l}=0$ is a singular boundary of the second kind, that is $\mathbf{M}\left(r_{l}\right)=\infty$. In addition, from its definition, $\varrho^{2}(r)=O\left|r-r_{l}\right|^{0}$ and based on the fact that $\mathscr{U}(r)$ is bounded around $r_{l}$, it follows that $\mathbf{M}(r)=O\left|r-r_{l}\right|^{-1}$ as $r \rightarrow r_{l}^{+}$; so the diffusion and drift exponents of $r_{l}$ are given by, respectively, $\mathbb{Q}_{l}=0, \beta_{l}=1$, and the character value of $r_{l}$ is

$$
\mathbb{C}_{l}=\lim _{r \rightarrow r_{l}^{+}} \frac{2 \mathbf{M}(r)\left(r-r_{l}\right)^{\mathbb{\beta}_{l}-\alpha_{l}}}{\varrho^{2}(r)}=1 .
$$

These quantities indicate that the left-hand boundary $r_{l}=0$ is an entrance. 
Similarly, the right-hand boundary $r_{r}=+\infty$ is a singular boundary of the second kind at infinity, that is $\mathbf{M}(+\infty)=\infty$. In this case, one can show that the diffusion and drift exponents of $r_{r}$ are given by, respectively $\mathbb{\alpha}_{r}=0$ and $\beta_{r}=1$. Note that $b-\varsigma=1+a \tau>0$ and $\lim _{x \rightarrow \infty} h^{\prime}(x)=$ 0 , hence $\mathbf{M}(+\infty)<0$. Therefore, the right-hand boundary $r_{r}=+\infty$ is repulsively natural. Since each of the two boundaries is either an entrance or repulsively natural, then a nontrivial stationary solution exists in $[0, \infty)$, which has the form $\mathrm{p}(r)=C r \exp \left(\frac{2 \tau^{2}}{a^{2} \sigma^{2}} \int_{0}^{r} \mathscr{U}(s) d s\right)$. Note that $\mathbb{p}(r)>0$ for $r \in(0, \infty)$, so the stationary probability is unique, see Kliemann (1987).

\section{A2. Proof of Theorem 4.2}

Based on Lin and Cai (2004), it is not difficult to verify the result that the boundaries $\pm \infty$ are singular boundaries of the second kind at infinity, that is $|\mathbb{M}( \pm \infty)|=\infty$ and the diffusion exponents and drift exponents of $\pm \infty$ are respectively $\alpha_{ \pm \infty}=0$ and $\beta_{ \pm \infty}=1$. In addition, $\mathbb{M}( \pm \infty) \lessgtr 0$. Therefore, $\pm \infty$ are repulsively natural, implying that there exists a nontrivial stationary solution in $(-\infty,+\infty)$. In fact, from the Fokker-Planck Equation, we know that the stationary probability is given by (4.6). Note that $\mathrm{p}(\psi)>0$ for all $\psi$, so the stationary probability density is unique.

\section{A3. Proof of Theorem 4.3}

It is obvious that $\psi=0$ is one of the solutions to (4.7) for any of the cases considered. In the following, we only consider the case $\psi \in(-\infty, 0)$ and a similar reasoning holds for the case $\psi \in(0,+\infty)$. Let $S(\psi)=-\frac{2 \psi\left(1-h^{\prime}(\psi)\right)^{2}}{a \sigma^{2}}$. By the assumptions on $h$, we know that $h^{\prime \prime}(\psi)$ is a concave function on $\left(x_{1}, 0\right)$ and $\lim _{\psi \rightarrow-\infty} h^{\prime \prime}(\psi)=\ell$ where $0 \leq \ell<+\infty$. In addition, $\lim _{\psi \rightarrow-\infty} S(\psi)=+\infty, S^{\prime}(\psi)<0$ for $\psi \in(-\infty, 0), S^{\prime \prime}(\psi)>0$ for $\psi \in\left(x_{1}, 0\right)$. When

$$
h^{(3)}(0)>-\frac{2}{a \sigma^{2}} \quad \text { and } \quad b<1-\sqrt{-\frac{h^{(3)}(0) a \sigma^{2}}{2}},
$$

then $S^{\prime}(0)<h^{(3)}(0)<0$. By the convexity and concavity of $S(\cdot)$ and $h^{\prime \prime}(\cdot)$ in $\left[x_{1}, 0\right)$ and monotonicity of $h$ in $\left(-\infty, x_{1}\right)$, there is no solution of $(4.7)$ on $(-\infty, 0)$. When (A.1) is satisfied, (4.7) has a unique solution $\psi=0$ on $(-\infty,+\infty)$ and $\mathbb{p}^{\prime \prime}(0)=\frac{N}{a \sigma}\left(-h^{(3)}(0)-\frac{2(1-b)^{2}}{a \sigma^{2}}\right)<0$, implying that $\psi=0$ is the maximum point of $\mathbb{p}(\cdot)$.

When $b>1-\sqrt{-h^{(3)}(0) a \sigma^{2} / 2}$, then $S^{\prime}(0)>h^{(3)}(0)$. Therefore, there exists a solution of $(4.7)$ in $(-\infty, 0)$, denoted by $\psi_{1}^{*}$. To demonstrate the uniqueness of the solution of $(4.7)$ in $(-\infty, 0)$, we consider the following two cases:

(i) If the solution $\psi_{1}^{*}$ is on $\left[x_{1}, 0\right)$, by the concavity of $h^{\prime \prime}(\cdot)$ and convexity of $S(\cdot)$ in $\left[x_{1}, 0\right)$, there is a unique solution of $(4.7)$ on $\left[x_{1}, 0\right)$. On the other hand, on $\left(-\infty, x_{1}\right), h^{\prime \prime}(\cdot)$ is monotonically increasing and $S(\cdot)$ is monotonically decreasing. Hence, there is no other solution on $(-\infty, 0)$.

(ii) When $\psi_{1}^{*} \in\left(-\infty, x_{1}\right)$, there is no solution of (4.7) on the interval $\left[x_{1}, 0\right)$, otherwise there is a contradiction with the case (i). With the monotonicity of $h^{\prime \prime}(\cdot)$ and $S(\cdot)$ on $\left(-\infty, x_{1}\right)$, there is only one solution $\psi_{1}^{*}$ on $(-\infty, 0)$. 
In addition, when $b>1-\sqrt{-h^{(3)}(0) a \sigma^{2} / 2}$, we have $\mathbb{p}^{\prime \prime}(0)>0$. So $\psi=0$ is the minimum point of $\mathbb{p}(\cdot)$. We note that at $\psi_{1}^{*}$, it must be the case that

$$
\left.h^{(3)}(\psi)\right|_{\psi_{1}^{*}}>\left.\left(\frac{-2 \psi\left(1-h^{\prime}(\psi)\right)^{2}}{a \sigma^{2}}\right)^{\prime}\right|_{\psi_{1}^{*}} .
$$

Otherwise, by $\lim _{\psi \rightarrow-\infty} h^{\prime \prime}(\psi)=\ell(<+\infty)$ and $\lim _{\psi \rightarrow-\infty} S(\psi)=+\infty$, there must be another solution $\widetilde{\psi}\left(<\psi_{1}^{*}\right)$ of $(4.7)$, which is a contradiction of the uniqueness of the solution of $(4.7)$ on $(-\infty, 0)$. Then, through (4.7) and (A.2)

$$
\begin{aligned}
\left.\mathbb{p}^{\prime \prime}(\psi)\right|_{\psi=\psi_{1}^{*}} & =\left[-\frac{h^{(3)}(\psi)}{a \sigma}+\frac{6 \psi h^{\prime \prime}(\psi)\left(1-h^{\prime}(\psi)\right)}{a^{2} \sigma^{3}}-\frac{2\left(1-h^{\prime}(\psi)\right)^{2}}{a^{2} \sigma^{3}}\right. \\
& \left.+\frac{4 \psi^{2}\left(1-h^{\prime}(\psi)\right)^{3}}{a^{3} \sigma^{5}}\right]\left.N \exp \left(\int_{0}^{\psi}-\frac{2 y\left(1-h^{\prime}(y)\right)}{a \sigma^{2}} d y\right)\right|_{\psi=\psi_{1}^{*}}<0 .
\end{aligned}
$$

Therefore, $\psi_{1}^{*}$ is a maximum point of $\mathbb{p}(\cdot)$.

\section{References}

Anderson, S. (1989), 'Evidence on the reflecting barriers: new opportunities for technical analysis?', Financial Analysts Journal 45(3), 67-71.

Andronov, A., Vitt, A. and Khaikin, S. (1966), Theory of Oscillators, Pergamon Press.

Arnold, L. (1998), Random Dynamical Systems, Springer-Verlag.

Arnold, L., Sri Namachchivaya, N. and Schenk-Hoppé, K. R. (1996), 'Toward an understanding of stochastic Hopf bifurcation: a case study', International Journal of Bifurcation and Chaos 6(11), 1947-1975.

Beja, A. and Goldman, M. (1980), 'On the dynamic behaviour of prices in disequilibrium', Journal of Finance 35, 235-247.

Böhm, V. and Chiarella, C. (2005), 'Mean-variance preferences, expectations formation, and the dynamics of random asset prices', Mathematical Finance 15(1), 61-97.

Böhm, V. and Wenzelburger, J. (2005), 'On the performance of efficient portfolios', Journal of Economic Dynamics and Control 29, 721-740.

Boswijk, H., Hommes, C. and Manzan, S. (2007), 'Behavioral heterogeneity in stock prices', Journal of Economic Dynamics and Control 31, 1938-1970.

Brenan, K. E., Campbell, S. L. and Petzold, L. R. (1989), Numerical Solution of Initial-Value Problems in Differential-Algebraic Equations, North-Holland.

Brock, W. and Hommes, C. (1997), 'A rational route to randomness', Econometrica 65, 10591095. 
Brock, W. and Hommes, C. (1998), 'Heterogeneous beliefs and routes to chaos in a simple asset pricing model', Journal of Economic Dynamics and Control 22, 1235-1274.

Brock, W., Hommes, C. and Wagener, F. (2005), 'Evolutionary dynamics in financial markets with many trader types', Journal of Mathematical Economics 41, 7-42.

Chiarella, C. (1992), 'The dynamics of speculative behaviour', Annals of Operations Research 37, 101-123.

Chiarella, C., Dieci, R. and He, X. (2009), 'Heterogeneity, market mechanisms, and asset price dynamics', in T. Hens and K. R. Schenk-Hoppé, eds, "Handbook of Financial Markets: Dynamics and Evolution", in the series of Handbooks in Finance (W. Ziemba, eds), Elsevier. Chapter 5, 277-344.

Chiarella, C., He, X. and Hommes, C. (2006a), 'A dynamic analysis of technical trading rules in financial markets', Journal of Economic Dynamics and Control 30, 1729-1753.

Chiarella, C., He, X. and Hommes, C. (2006b), 'Moving average rules as a source of market instability', Physica A 370, 12-17.

Chiarella, C., He, X. and Zheng, M. (2008), 'The stochastic dynamics of speculative price', Quantitative Finance Research Centre, University of Technology, Sydney, working paper 208.

Chiarella, C., He, X., Wang, D. and Zheng, M. (2008), 'The stochastic bifurcation behaviour of speculative financial marekts', Physica A 387, 3837-3846.

Diks, C. and Wagener, F. (2006), 'A weak bifurcation theory for discrete time stochastic dynamical system', CeNDEF Working Paper 06-04, University of Amsterdam.

Feller, W. (1952), 'The parabolic differential equation and the associated semigrougps of transformations', Annals of Mathematics 55, 468-519.

Föllmer, H. (1974), 'Random economics with many interacting agents', Journal of Mathematical Economics 1, 51-62.

Föllmer, H., Horst, U. and Kirman, A. (2005), 'Equilibria in financial markets with heterogeneous agents: a probabilistic perspective', Journal of Mathematical Economics 41, 123 155 .

Grasman, J. (1987), Asymptotic Methods for Relaxation Oscillations and Applications, Vol. 63, Applied Mathematical Sciences, Springer-Verlag.

He, X. and Li, Y. (2007), 'Power law behaviour, heterogeneity, and trend chasing', Journal of Economic Dynamics and Control 31, 3396-3426.

Hens, T. and Schenk-Hoppé, K. R. (2005), 'Evolutionary stability of portfolio rules in incomplete markets', Journal of Mathematical Economics 41, 43-66. 
Hommes, C. (2002), 'Modeling the stylized facts in finance through simple nonlinear adaptive systems', Proceedings of National Academy of Science of the United States of America 99, 7221-7228.

Hommes, C. (2006), Heterogeneous agent models in economics and finance, in L. Tesfatsion and K. Judd, eds, 'Agent-based Computational Economics', Vol. 2 of Handbook of Computational Economics, North-Holland, chapter 23, pp. 1109-1186.

Hommes, C. and Wagener, F. (2009), 'Complex evolutionary systems in behavioral finance', in T. Hens and K. R. Schenk-Hoppé, eds, "Handbook of Financial Markets: Dynamics and Evolution", in the series of Handbooks in Finance (W. Ziemba, eds), Elsevier. Chapter 4, 217-276.

Horst, U. and Rothe, C. (2008), 'Queuing, social interactions, and the microstructure of financial markets', Macroeconomic Dynamics 12, 211-233.

Horst, U. and Wenzelburger, J. (2007), 'On non-ergodic asset prices', Economic Theory 34, 207234.

Khas'minskii, R. (1963), 'The behaviour of a self-oscillating system acted upon by slight noise', Journal of Applied Mathematics and Mechanics, English translation of Prikladnaya Matematika i Mekhanika 27, 683-687.

Kliemann, W. (1987), 'Recurrence and invariant measures for degenerate diffusions', Annals of Probability 15(2), 690-707.

LeBaron, B. (2006), Agent-based computational finance, in L. Tesfatsion and K. Judd, eds, 'Agent-based Computational Economics', Vol. 2 of Handbook of Computational Economics, North-Holland, chapter 24.

Lee, C. and Swaminathan, B. (2000), 'Price momentum and trading volume', Journal of Finance 55, 2017-2069.

Lin, Y. and Cai, G. (2004), Probabilistic Structural Dynamics, McGraw-Hill, New York.

Lux, T. (1995), 'Herd behaviour, bubbles and crashes', The Economic Journal 105, 881-896.

Lux, T. (1997), 'Time Variation of Second Moments from a Noise Trader/Infection Model', Journal of Economic Dynamics and Control 22, 1-38.

Lux, T. (1998), 'The socio-economic dynamics of speculative markets: interacting agents, chaos, and the fat tails of return distributions', Journal of Economic Behavior and Organization 33, 143-165.

Lux, T. (2009), 'Stochastic behavioral asset-pricing models and the stylized facts', in T. Hens and K. R. Schenk-Hoppé, eds, "Handbook of Financial Markets: Dynamics and Evolution", in the series of Handbooks in Finance (W. Ziemba, eds), Elsevier. Chapter 3, $161-215$. 
Mao, X. (1997), Stochastic Differential Equations and Applications, Horwood Publishing.

Pagan, A. (1996), 'The econometrics of financial markets', Journal of Empirical Finance 3, 15102.

Rheinlaender, T. and Steinkamp, M. (2004), 'A stochastic version of Zeeman's market model', Studies in Nonlinear Dynamics and Econometrics 8, 1-23.

Wagenmarkers, E., Molenarra, P., Grasman, R., Hartelman, P. and van der Maas, H. (2005), 'Transformation invariant stochastic catastrophe theory', Physica D 211, 263-276.

Yurkevich, V. D. (2004), Design of Nonlinear Control Systems with the Highest Derivative in Feedback, Vol. 16, Series on Stability, Vibration and Control of Systems, World Scientific.

Zeeman, E. (1974), 'On the unstable behaviour of stock exchanges', Journal of Mathematical Economics 1, 39-49. 